\title{
BP Neural Networks Algorithm Applied in Electric Load Prediction
}

\author{
Duan Ping \\ Department of Information Engineering \\ Hubei Urban Construction Vocational And Technological College, Wuhan, China
}

\begin{abstract}
After analyzing the meaning and ways of electric system load prediction, this essay illustrates the general theory of the BP neural networks and study the load prediction methods based on BP neural networks. It analyzes the neural networks sample value methods, performs encumbrance for historical data, then, it preprocesses the load data. The accuracy of the load prediction results is closely related to the preprocessing of the load data. The data processed correctly will produce distinct effect on the accuracy of the prediction results. Contrarily, inputting the load data unprocessed into the neural networks will result in prediction failure. This essay designs the structure of the algorithm of BP neural networks. At last, it analyzes the data of the case study of electric load prediction.
\end{abstract}

Keywords: BP neural networks; electric load; prediction; algorithm;

\section{Introduction}

The electric load prediction is, under the condition of considering some important elements (such as system running features, increase-volume decision, natural condition and social influence), a math method to study or use a set of system to process the past and future load. Under the condition of satisfying certain accuracy requirements, determine the load value at some specific moment in the future. Elec- tric load prediction is one of the important works for the electric power supply department. Accurate load prediction can help arrange economically and reasonably the start and the stop of the inner generating unit of the power grids, keep the power grids safe and stable, reduce unnecessary revolving reserve capacity; can help arrange reasonably the maintenance schedule for the unit, ensure social normal production and human life, effectively reduce generation cost and increase economic and social benefits. The load prediction result is also helpful for deciding the installation of the new generating unit in the future, for deciding the size, location and time of the installed capacity, for deciding the increase-volume and the improvement of the power grids, for deciding the construction and the development of the power grids. Therefore, the level of the electric load prediction work has become one of the distinct marks to measure whether the managements of an electric power enterprise modernizes. Especially now our country's electric industry is developing at an unprecedented rate, how to solve the problem of electric load prediction has become a faced important and hard task. Electric frequency key technology, many projects the power grids plan are all for the study on the electric load prediction and market demand prediction and so on. So it can be seen that how to make full use of the existing data, establish correct prediction theory and methods and establish corresponding prediction models, increase 
prediction accuracy has become one of the study subjects that must not be overlooked on electric prediction in electric system.

\section{The basic concept on BP Artificial neural networks}

This essay adopts the BP artificial neural networks to perform electric load prediction. The back propagation neural networks are known for that the error signal anti-propagates. It chooses to use the forward neural networks. There are $3 \mathrm{lev}$ els in the networks model: input level, output level and middle level. Basically, error back propagation study consists of two procedures: one forward passing and one backward passing. In the forward passing, the vector is input through input level. Then, it forward propagates through the neural networks by one level after another. At last, it produces an output as the actual response to the networks. During the process of forward propagation, the connection weight value between networks is changeless. Another procedure is back propagation. During the procedure of back propagation, all the connection weights between neurons shall adjust according to the error correction rules, in which, error signal shall be obtained from the difference between the networks response gained from the process of forward propagation and the target response. When the error reaches certain requirement, the training of

The convergence rate of the basic back propagation algorithm is very slow. It will take several days even several weeks to perform load prediction and training by using the basic back propagation algorithm. Therefore, it is the key problem to increase the convergence rate of the algorithm. In general, there are two ways to raise the convergence rate. The first class includes using the heuristic information technology. This is from the study on the specific performances of the error curve of the standardized back propagation algorithm. These heuristic technologies include the alterable learning rate, using the momentum and altering the rate variable. Another class is the standardized quantitative value optimization technique. In fact, it is just a problem of the quantitative value optimization for the training forward neural networks to reduce means square error. Because the quantitative value optimization has been an important study subject for four or five decades, it is more reasonable to choose the fast training algorithm from the large quantity of the existing quantitative value optimization techniques, mainly including the conjugate gradient algorithm and Levenbegr-Marquardt algorithm (an improved Newton algorithm) and so on.

\section{Application of algorithm of the BP nerual networks in electric load prediction}

Before using the neural networks to perform load prediction, it is a very important step to prepare for the historical load data. The data processed correctly will produce distinct effect on the accuracy of the prediction results. Contrarily, inputting the load data unprocessed into the neural networks will result in prediction failure.

The test data is a part of the input data, which is only used to test how the neural networks adapt to the new data. The test data is not used when training the neural networks but used to test how big the error is in the future networks applications when the training ends.

\subsection{The preprocessing of the load data}

The load data which has been arranged shall be processed properly before being input to the neural networks to make the data specification meet the requirement of the neural networks. For example, nor- 
malized load data and text data shall be converted to numerical data and so on. The weather factor and holiday factor usually are text data, while the input layer of the neural networks can only receive numerical data. Therefore, it is necessary to convert these text data before being input to the neural networks. Only after the text data converted to numerical data can they be recognized by the neural networks. For example, the low, medium, high text form shall be expressed as the numerical expression as $\{1,0,0\},\{0,1,0\}$ and $\{0,0,1\}$ respectively. Additionally, because the data range of the training samples can be restricted by the neurons of the artificial neural networks, it is necessary to perform the training samples normalization processing. For the numerical data in the input layer, convert it to the value in $[-1,1]$. The conversion formula shall be as follows:

$$
\begin{aligned}
& S F=\left(S R_{\max }-S R_{\min }\right) /\left(X_{\max }-X_{\min }\right) \\
& X_{p}=S R_{\min }+\left(X-X_{\min }\right) \times S F
\end{aligned}
$$

Here, $X$ is the actual value of numerical data in some column. $X_{\text {min }}$ is the minimum value of the actual quantitative value in this column. $X_{\max }$ is the maximum value of the actual quantitative value in this column. $S R_{\min }$ is the lower limit value after normalization. $S R_{\max }$ is the upper limit value after normalization. $S F$ is the normalization factor. $X_{p}$ is the quantitative value to be processed. While it is necessary to perform approximation processing the target numerical data in the output layer according to the range of the activation function.

\subsection{Structure design of the artificial neural networks}

For the choice of the input variables of the neural networks, the weather and hol- iday factors which have much impact on the load can be considered first. Input this plan to some neural networks to perform certain times training. Then, gradually add some other influence factors to perform the same times training and stop until the added factors improve not much networks error.

The experiences have shown that for the problem of distinguishing the boundary of the small-sized network, two-layer hidden layer is not more superior to single hidden layer. Therefore, at present, people consider that just a hidden layer will be enough for the classification of the binary system or the boundary distinguishing problem. But if it is required that the output is the continuous function of the input, it will be required to adopt two hidden layers or different activation functions. Sometimes, even is the continuous output, one hidden layer can satisfy the requirement. This depends on the nature of the problem. Please note that under the condition of linearly separable, the hidden layer is unneeded.

The choice of the number of the nodes in each layer of the neural networks based on the BP algorithm has great impact on the performances of the networks. So, it is necessary to choose proper number of the nodes within layers. Generally, how many hidden layers a multi-layer network needs, how many hidden units a layer needs, it depends on the use of the networks. But, this is not the only determination. Because adopting different internal images, the needed internal units are various. Therefore, it is a very complex problem to choose the number of the hidden layer units.

The neural networks having single hidden layer will get the satisfied results. The decision domain of the output of this kind of single hidden layer is usually convex domain. Following introduce several estimation value methods to choose 
the number of the single hidden layer nodes.

If the output nodes is $M_{1}$,

Lippmann considers the maximum number of the hidden layer nodes is $M_{1}(N+1)$.

Kuarycki considers the maximum number of the hidden layer nodes is $M_{1} \times 3$.

A.J.Maren considers that, for the small-sized networks, when the input nodes are more than the output nodes, the best number of the hidden layer nodes equals to the geometric average of the input and output nodes, namely, $\left(M_{1} \times N\right)^{1 / 2}$.

These reference formulas above, for the classifier, because its input and output basically are two-valued function, if the output is two-valued images or features, the output is 0,1 and so on, the model of the perceptron can be used as reference. For the BP networks performing the approximation of functions, the number of the units in the middle layer is related to the accuracy of the function of approximation and the fluctuation situation of the function itself. For example, it is required the number of the approximation of the trigonometric function or the number of the terms of the multinomial increase, therefore, the number of the hidden units shall increase. Similarly, the function fluctuates more in the closed interval, the higher frequency of the trigonometric function shall be required, thus, terms are more. In this way, the number of the hidden units is more. This is only rude explanation, there is no very strict relation. Actually, because the hidden unit itself also can extend to the sum of many trigonometric functions or multinomial, so the number of the hidden units is also related to the nature of the approximation of the function itself.

\section{Conclusion}

The accuracy of the load prediction results is closely related to the preprocessing of the load data. The data processed correctly will produce distinct effect on the accuracy of the prediction results. Contrarily, inputting the load data unprocessed into the neural networks will result in prediction failure. This essay designs the structure of the algorithm of BP neural networks. At last, it analyzes the data of the case study of electric load prediction.

\section{References}

[1] Liu Chen hui. "Electric System Load Forecasting Theory and Methods"., Harbin Industry University Press, 2007

[2] Xin Kaiyuan, Cao Shuhua. "Several Methods of Electric System Short Time Load Prediction", Electric Information, 2003 (3), 8,14

[3] Niu Xiaodong, Cao Shuhua, "Electric Load Prediction Technology and Applications", China Electric Press, 2008

[4] H.C.Wu, C.N.Lu, "Automatic Fuzzy Model Identification For Short-Term Load Forecasting", IEE Proc.-Gener. Transm. Distrib., Vol.146. No.5, Sep. 2009, 477 482

[5] Andrew P. Douglas, Arthur M. Breipohl, Fred N. Lee, Rambabu Adapa, "The Impacts Of Temperature Forecast Uncertainty On Bayesian Load Forecasting", IEEE Transactions on Power System, Vol.13.No.4, Nov.2008, 1507-1513. 